\title{
Acousto-optic modulation in a microstructured plastic optical fibre Bragg grating
}

\author{
L. Bilro*a, C.A.F. Marques ${ }^{a}$, L. Khan ${ }^{b}$, R. A. Oliveira ${ }^{c}$, D. J. Webb ${ }^{b}$, R. N. Nogueira ${ }^{a}$ \\ anstituto de Telecomunicações, Campus Universitário de Santiago, 3810-193 Aveiro, Portugal \\ ${ }^{\mathrm{b}}$ Aston Institute of Photonic Technologies, Aston University, Birmingham, B4 7ET, UK \\ ${ }^{c}$ Volvo 3P-PD Advanced Engineering - Brazil, Av. Juscelino K. de Oliveira, 2600, \\ 81.260-900, Curitiba, Paraná, Brazil
}

\begin{abstract}
The present work addresses the control of the mPOF Bragg grating spectrum properties through acousto-optic modulation. For the first time, the interaction of a flexural acoustic wave, generated by longitudinal excitation of different frequencies, with the Bragg grating will be presented. Also it will be demonstrated the quasi linear relationship between PZT load and maximum reflected power/ $3 \mathrm{~dB}$ bandwidth of the reflected spectrum.
\end{abstract}

Keywords: acoustic-optic effect, fibre Bragg gratings, flexural and longitudinal modes, microstructured plastic optical fibre.

\section{INTRODUCTION}

Among the different technologies available today, fibre Bragg gratings (FBG) are pointed out as an interesting device for performing all-optical signal processing and for sensing applications. Most FBGs referenced in the literature are inscribed in silica fibres, however a major drawback of using silica is that only a few percent of elongation is achieved. This fact motivated the scientific community to investigate the possibility of inscribing FBGs in new types of fibres, namely in polymer optical fibre (POF). The properties of POF are quite different to those of silica and offer some significant potential advantages such as easy non-skilled handling and ruggedness. The first FBG in SI-POF was produced in 1999 [1]. The Young modulus of polymeric materials (mainly PMMA) is approximately 25 times less than that of silica and POF can withstand and measure much higher strains. Thus higher shifts of Bragg wavelength are obtained for FBG-POF than with silica fibre using temperature [2], strain [3] or bending variations.

POF development also focused on microstructured designs, with considerable progress in the manufacture of microstructured POF (mPOF) [4]. The geometry of this type of fiber provides different properties compared to a stepindex fiber, such as an endlessly single-mode, air-guiding operation, and the ability to expose the electric field of the guided mode to substances contained within the holes. The first FBG inscribed onto mPOF was obtained by Dobb et al., using a low-power HeCd laser at $325 \mathrm{~nm}$ [5]. The resulting Bragg grating had a $\lambda_{\mathrm{B}}$ of $1536 \mathrm{~nm}$ and a length of $10 \mathrm{~mm}$. For a $3 \%$ maximum deformation, this research group obtained a $\lambda_{B}$ variation of $31 \mathrm{~nm}$.

On the other hand, the acousto-optic effect is an important, fast and accurate mechanism that can be used to change and control several of the properties of FBGs. It has been successfully applied in the design and construction of various low insertion loss all-fibre devices such as a tunable dispersion compensator, tunable optical notch filter $[6,7]$ and complex Bragg grating writing [8], among others. Also, using a LPG, a viscosity and density sensor was reported [9].

Due to the mechanical properties of polymers it is expected that the acousto-optic effect in POF will be more noticeable because acoustic impedance will be lower; improving the performance of the silica based existing devices. To our knowledge, this is the first report on the control of the mPOF Bragg grating spectrum properties through acousto-optic modulation. The interaction of a flexural acoustic wave, generated by longitudinal excitation, with the Bragg grating will be presented.

*lucia.bilro@ua.pt; Phone: +351 234377 900, Fax: +351 234377901. 


\section{EXPERIMENTAL SET-UP}

The grating used in these experiments was inscribed in a PMMA based few moded mPOF with the phase mask technique, using a $325 \mathrm{~nm} \mathrm{HeCd}$ laser. The Bragg wavelength was set in $1570 \mathrm{~nm}$, the grating spectrum width and length were about $5 \mathrm{~nm}$ and $2 \mathrm{~mm}$, respectively. The grating was connectorized to a silica fibre using UV curable glue.

The acousto-optic (AO) modulator used is composed of a piezoelectric transducer (PZT), a silica horn and the mPOF containing the FBG. The silica horn was characterized by a length of $50.6 \mathrm{~mm}$, a base diameter of $5 \mathrm{~mm}$, a tip diameter of $1 \mathrm{~mm}$ and a central hole diameter of $125.5 \mu \mathrm{m}$. The end of the fibre is fixed to a holder, which enables the generation of an acoustic standing wave. The set was swept over the range from $1 \mathrm{kHz}$ to $1 \mathrm{MHz}$.

As can be seen in Figure 1, the AO modulator is connected to an arbitrary function generator and the reflected spectra were monitored by an interrogator system (SM125-500 MicronOptics), with wavelength accuracy of $1 \mathrm{pm}$.

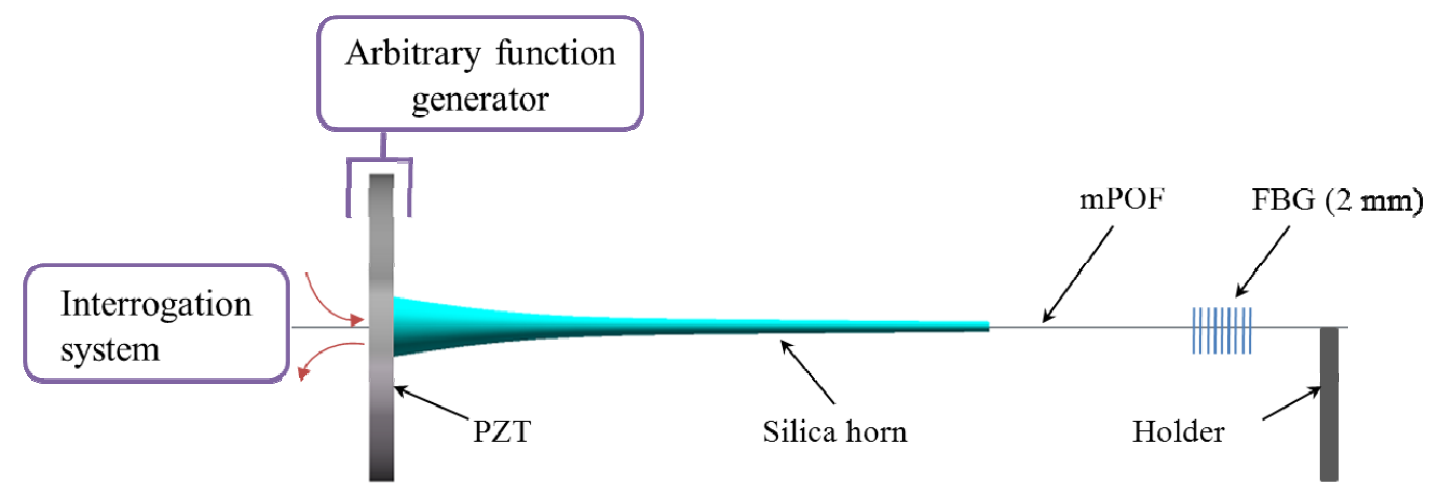

Figure 1. Experimental set-up used to excite acoustic waves in the silica horn structure.

\section{RESULTS}

Depending on the applied acoustic frequency, different modes can be excited in the fibre, namely flexural, longitudinal or hybrid. A detailed study of the natural vibration modes of the mechanical structure can be found in [10]. The study showed that low frequency resonant modes generate flexural acoustical waves that cause bends in the fiber.

Figure 2a) shows the result for the acoustic excitation of $77 \mathrm{kHz}$. It can be seen that a decrease in the peak and a broadening of the reflected spectrum occur when the acoustic wave is turned on. This result can be explained considering the coupling mechanism of the propagating modes. The lower reflectivity of the grating corresponds to a phase mismatching and a consequent coupling between the fundamental mode and radiation modes. Although is not as strong as for $77 \mathrm{kHz}$, in Figure 2b) the same effect can be observed for an acoustic excitation of $193 \mathrm{kHz}$. However, it is worth to note a small wavelength shift when the excitation is applied to the PZT. This shift occurs because the vibration mode is hybrid. When a hybrid mode is found at a low frequency the flexural vibration is prevailing [10]. Nevertheless, the longitudinal acoustic wavelength is higher than the FBG length and so a compression and/or rarefaction strain field with less than on period of the standing wave is applied in the grating. The result of a compression strain field is a small blue shift of $\lambda_{\mathrm{B}}$. Such effects are also noted for $19 \mathrm{kHz}, 178 \mathrm{kHz}$ and $302 \mathrm{kHz}$. In Figure 2c), an acoustic excitation of $214 \mathrm{kHz}$ results in the onset of two peaks in the optical spectrum. This may be a result of the grating being in the transition between compression to rarefaction strain fields, corresponding to both blue and red shifting of the $\lambda_{\mathrm{B}}$.

With respect to high frequencies, it was noticed that for frequencies higher than $302 \mathrm{kHz}$ (the longitudinal regime) no effects on the FBG spectrum were observed. The explanation for this absence of grating modulation is that the longitudinal displacement of the grating planes does not occur because of the small grating length. In fact, the observation of the acousto-optical modulation in a $2 \mathrm{~mm}$ grating is already a significant result because using a FBG in silica fibre this would not be possible (the effect is only noticed with grating lengths in the order of $\mathrm{cm}$ ). 


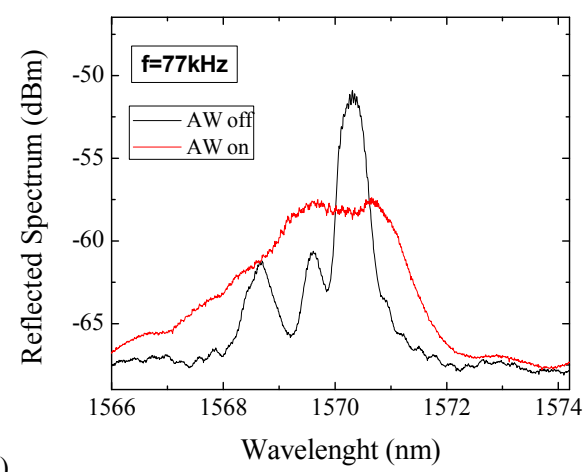

a)

Figure 2. Spectrum behavior for "ON" and "OFF" of the acoustic wave with a frequency of a) $77 \mathrm{kHz}, \mathrm{b}) 193 \mathrm{kHz}$, and

c) $214 \mathrm{kHz}$. PZT load was $20 \mathrm{~V}$.

Figure 3 shows the spectrum behavior of the grating when the applied PZT voltage varies from $0 \mathrm{~V}$ to $17 \mathrm{~V}$ at the acoustical excitation of $77 \mathrm{kHz}$. A reflectivity decrease is noticed with the increase of the applied PZT voltage. The relationship between the amplitude of the acoustic wave and the maximum reflected power becomes quasi-linear after the load is set at $3 \mathrm{~V}$. On the other hand, it is also possible to control the $-3 \mathrm{~dB}$ bandwidth of the reflected spectrum by tuning the applied PZT voltage. As the PZT load is increased the phase mismatching effect also increased.

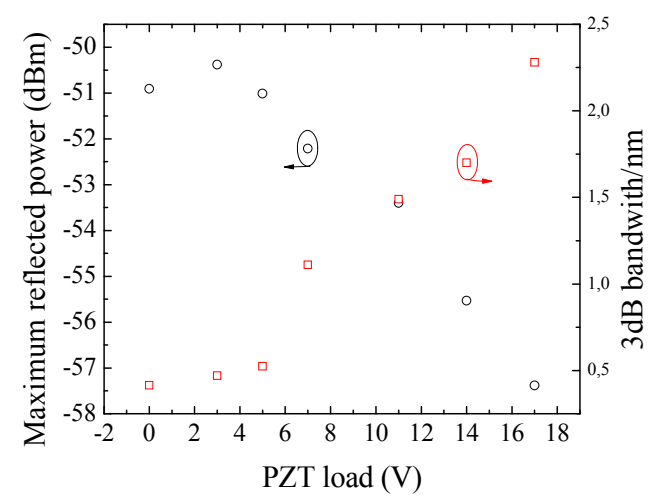

Figure 3. Spectrum properties for an acoustic wave with a frequency $77 \mathrm{kHz}$ and a PZT load range $0 \mathrm{~V}-17 \mathrm{~V}$.
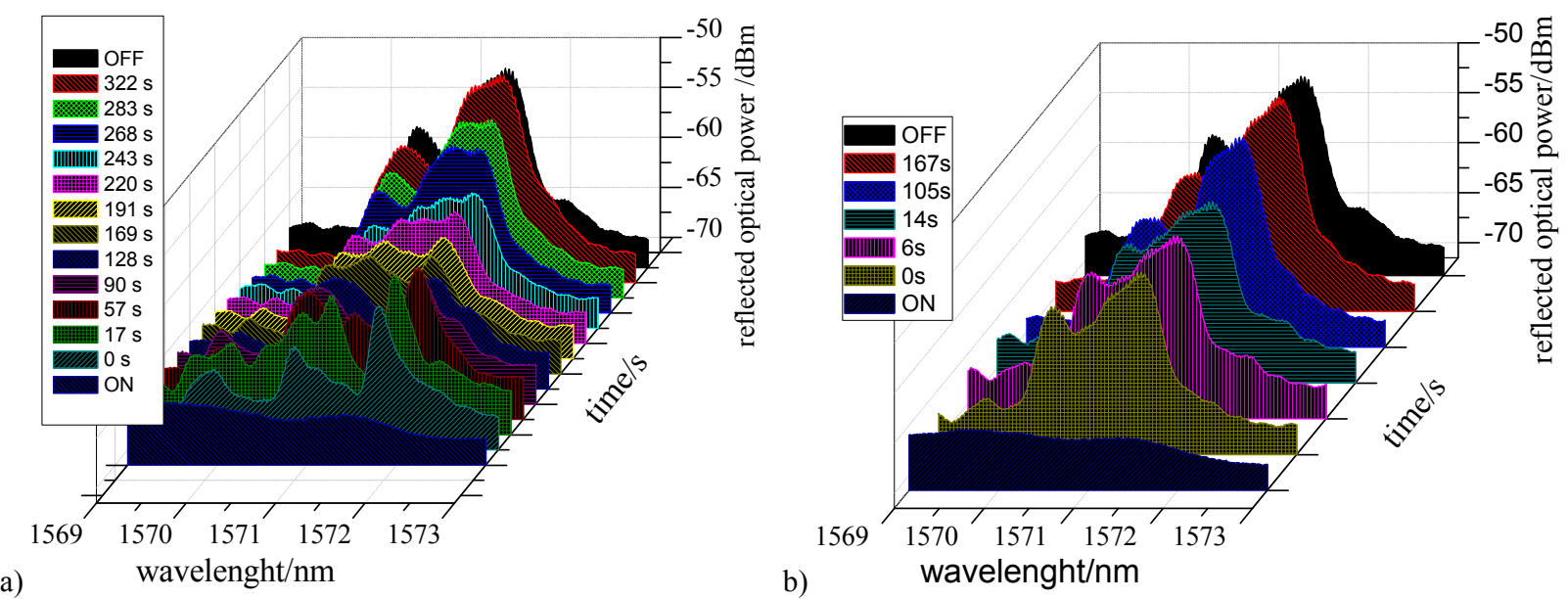

Figure 4. Regeneration of the FBG after an excitation of an acoustic wave with a frequency $77 \mathrm{kHz}$ during a) $35 \mathrm{~s}$ and b) $3 \mathrm{~s}$. PZT load was $20 \mathrm{~V}$. 
During the experiments it was noticed that for the frequency with the strongest acoustic effect on the properties of the reflected spectra, i.e. $77 \mathrm{kHz}$, the regeneration of the grating after turning off the acoustic wave is not instantaneous. As can be seen in Figure 4a), the regeneration of the grating lasted $322 \mathrm{~s}$ for a time of excitation of $35 \mathrm{~s}$. It seems that this phenomenon is the more effective the greater the excitation time. For a time of excitation of $3 \mathrm{~s}$ the regeneration time was evaluated as $167 \mathrm{~s}$. This delay observed will be addressed in more detail in future developments however it may be pointed out the viscoelastic properties of polymers as a possible explanation. The viscoelastic processes in polymers are absorption modes by which energy can be coupled into a material [11]. When the stimulus is stopped there will be internal molecular rearrangements during relaxation.

\section{CONCLUSIONS}

The presented report demonstrates that the excitation of an FBG written in MPOF through flexural acoustic waves can be attained. Results show that it is possible to dynamically control the properties of the grating spectra even with a grating length of $2 \mathrm{~mm}$. The obtained results open the possibility of using the device passively, as a temperature and strain sensor, and actively because adding a temporal element using acoustic waves, parameters that require dynamic assessment become accessible, such as viscosity and density. Future developments will be focused in the improvement of the theoretical understanding of the AOM phenomena in $\mathrm{MPOF}$ and the dynamic rheological properties of PMMA.

\section{ACKNOWLEDGMENTS}

The authors acknowledge the support from Fundação para a Ciência e a Tecnologia through the projects PTDC/CTM/101538/2008; PTDC/SAU-BEB/100650/2008; PTDC/EEA-TEL/114144/2009 and PTDC/EEATEL/122792/2010. Lúcia Bilro and Carlos Marques gratefully acknowledge the financial support received from the same institution, through fellowships, SFRH/BPD/78205/2011 and SFRH/BD/70661/2010, respectively.

\section{REFERENCES}

[1] Xiong, Z., Peng, G., Wu, B. and Chu, P., "Highly tunable Bragg gratings in single-mode polymer optical fibers," IEEE Photonics Technology Letters 11, 352-354 (1999).

[2] Liu, H. Y., Liu, H. B. and Peng, G. D., "Tensile strain characterization of polymer optical fibre Bragg gratings," Optical Communications 251, 37-43(2005).

[3] Liu, H. Y., Peng, G. D. and Chu, P., "Thermal tunning of polymer optical fiber Bragg gratings," IEEE Photonics Technology Letters 13, 824-826 (2001).

[4] Barton, G., van Eijkelenborg, M. A., Henry, G., Large, M. C. J. and Zagari, J., "Fabrication of microstructured polymer optical fibres," Optical Fiber Technology 10, 325-335 (2004).

[5] Dobb, H., Webb, D. J., Kalli, K., Argyros, A., Large, M. C., van Eijkelenborg, M. A., "Continuous wave ultraviolet light induced fiber Bragg gratings in few and single-mode microstructured polymer optical fibers," Optics Letters 30, 3296-3298 (2005).

[6] Marques, C.A.F., Oliveira, R.A., Pohl, A., Canning, J., Nogueira, R.N., "Control of the Properties of Fiber Bragg Gratings Based on the Accousto-Optic Effect," Proc European Conf. on Optical Communications - ECOC, Torino, Italy, pp. 1-3 (2010).

[7] Marques, C.A.F., Oliveira, R.A., Pohl, A., Canning, J., Nogueira, R.N., "Dynamic Control of a Phase-Shifted FBG through Acousto-Optic Modulation," Optics Communications 284(5), 1228 - 1231 (2011).

[8] Oliveira, R.A., Marques, C.A.F., Canning, J., Nogueira, R.N., Pohl, A., "Complex Bragg Grating Writing using Direct Modulation of the Optical Fibre with Flexural Waves," Applied Physics Letters 99(16), 161111-161111-3 (2011).

[9] Oliveira, R.A., Possetti, G. R. C., Marques, C.A.F., Neves, P.T., Bavastri, C. A., Kamikawachi, R. C., Fabris, J. L., Nogueira, R.N., Canning, J., Pohl, A., "Acousto-optic control of the LPG spectrum for sensing applications," Proc Optical Fiber Sensors Conf. OFS11-OFS21-223, Ottawa, Canada, 1-4 (2011).

[10] Oliveira, R. A., Neves, P. T. Jr, Pereira, J. T., Canning, J., and Pohl, A., "Vibration mode analysis of a silica hornfiber Bragg grating device," Optics Communications 283, 1296-302 (2010).

[11] Vachhani, N. A., "Using Narrowband Pulse-shaping to Characterize Polymer Structure and Dynamics - Deathstar GHz Spectroscopy,” MSc Thesis, Massachussetts Institute of Technology, 2005. 